\title{
RETROPERITONEAL CARCINOID
}

\author{
(Case Report)
}

\author{
Lt Col KAILASH CHAND *, Lt Col NK PANICKER ${ }^{+}$
}

MJAFI 1999; 55 : 167-168

KEY WORDS : Retroperitoneal tumors; Carcinoid

\section{Introduction}

$\mathrm{C}$ arcinoid tumours, unlike malignant neopldsm, are known to remain asymptomatic for years and undetected during life [1]. The clinical presentations of carcinoid, far under estimate their occurrence because significant number of cases remain asymptomatic. $85 \%$ of all carcinoid tumours develop in the gastrointestinal tract (GIT) and the appendix is the most common primary site [2]. Retroperitoneal carcinoids are extremely rare tumours. In a cumulative analysis of all types of carcinoid tumours from 195091, Modlin and Sandor[1] analysed 8305 cases originating from different sites and reported only 4 cases of carcinoids in the retroperitoneum accounting for just $0.05 \%$ of all the carcinoid tumours.

We encountered one such case recently, and were encouraged to highlight the available literature and induce awareness of this rare entity.

\section{Case Report}

A 55 years-old male reported with the complaints of a gradually increasing painless mass in front of left thigh of 7 years duration and discomfort in movements at the hip of 1 year duration. There was no history of trauma, sudden change in size of the mass or associated constitutional symptoms. On examination, there was a diffuse, nontender mass on the anterior surface of the left thigh extending from the groin towards knee. It was smooth surfaced, soft to firm in consistency with well defined margins, measured $25 \times 15 \mathrm{~cm}$ in size and adherent to the underlying structures. Flexion at the hip was restricted. A similar mass measuring $5 \times 4 \mathrm{cms}$ was also appreciated on deep palpation in left iliac fossa. There was pitting odema present over the left foot.

Fine needle aspiration of the mass in left iliac fossa and the thigh revealed a round cell tumour (Fig.1). Immunohistochemistry of the smears showed positive reactivity to cytokeratin and neuron specific enolase. biopsy of the tissue showed, tumour composed of small cells with scanty cytoplasm, having uniform central round or oval nuclei with regular margins. Nuclei were mitotically inactive. A few small glands with rossette like appearance, supported by delicate fibrovascular bands, were present (Fig.2). It was reported as carcinoid and the patient was investigated for the primary site in the abdomen.
Upper and lower gastrointestinal endoscopy were inconclusive. Chest radiograph and ultrasound of the abdomen were non-contributory. Computed tomography (CT) scan of pelvic region revealed a large mass over the vascular channels in the left pelvis extending over the thigh. The patient was operated for the purpose of debulking. The operation findings revealed that the tumour

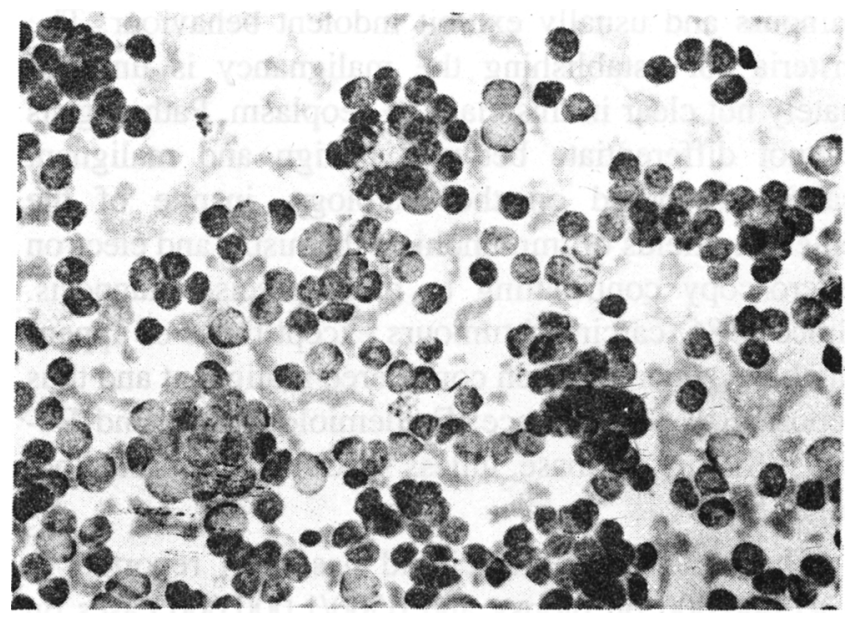

Fig. 1: $40 \mathrm{x}$ fine needle aspiration cytology showing monomorphic round cell population of carcinoid having scanty cytoplasm and large round central nuclei.

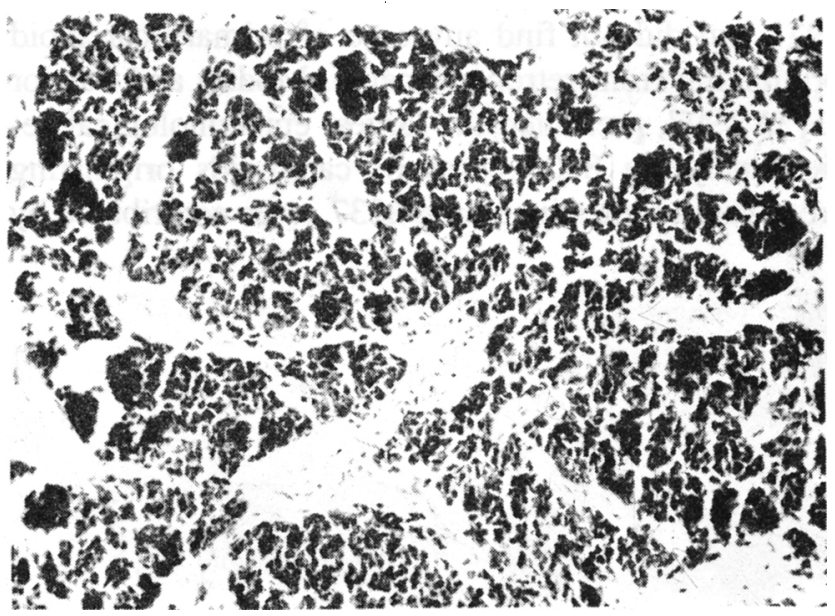

Fig. 2: $10 \mathrm{x}$ carcinoid tumour showing monotonous sheets of cells having uniform round nuclei arranged in small glands and rosette like pattern.

\footnotetext{
${ }^{*}$ Classified Specialist (Pathology), Military Hospital (CTC), Pune $411040 .^{+}$Associate Professor of Pathology, Armed Forces Medical College, Pune 411 040. (Presently Professor of Pathology, D.Y. Patil Medical College for Women, Pune).
} 
was firmly adherent to the femoral blood vessels extending upto the bifurcation of aorta. It was apparently arising from the retroperitoneum and was not adherent to the gut. The tumour over the thigh was excised but the retroperitoneal tumour had to be left behind due to considerable bleeding during the operation.

\section{Discussion}

The term carcinoid is derived to describe, a cancer like tumour that appeared to behave less aggressively than classic carcinoma. Later on, like other tumours, the malignant potential of these tumours was realised as upto $50 \%$ cases of the intestinal carcinoids presented with widespread metastasis. Few patients developed signs and symptoms at metastatic sites, and the primary site remained elusive despite extensive investigations $[2,4]$.

Carcinoids belong to the group of neuroendocrine tumours and usually exhibit indolent behaviour. The criteria for establishing the malignancy is unfortunately not clear in this class of neoplasm. Pathologists cannot differentiate between benign and malignant carcinoids based on the histology, inspite of the emerging fields of immunohistochemistry and electron microscopy contributing to more precise diagnosis. Since 1986, carcinoid tumours except those of appendix have generally been considered malignant and thus reported to Surveillance, Epidemiology and End Results (SEER) database, unless specifically reported benign [5].

The incidence of carcinoid has been reported 7$13 / 1,000,000$ on biopsy and $6500 / 1,000,000$ cases on autopsy[3]. Godwin [6] in 1975, analysed 2837 cases of carcinoid tumour identified by End Result Group and Third National Cancer Survey between 1950 to 1971 and did not find any case of primary carcinoid originating from retroperitoneum. Modlin and Sandor [1] in 1997, presented the largest epidemiological series analysing 8305 cases of carcinoids originating from various sites including 2837 cases contributed by
Godwin and 5468 cases identified by SEER program of National Cancer Institute from 1973-91. The most frequent site of carcinoid in the series was, GIT (73.7\%) followed by bronchopulmonary $(25.1 \%)$, appendix (18.3\%) and rectum (12.6\%). Carcinoid originating from retroperitoneum was listed in only 4 out of 8305 cases $(0.05 \%)$. Other rare primary sites being oesophagus, liver, cervix uteri and larynx $(<0.05 \%)$.

The incidence of carcinoid is increasing due to increased awareness of the entity and introduction of improved diagnostic procedures. Localisation of tumours particularly from unknown primary site may be helped by ultrasound studies, high resolution CT scanning and high selective angiography. Radioisotopes tags to specific antibodies and peptides, intraoperative ultrasound and magnetic resonance image scanning are the other investigations useful in the localisation of primary tumour [7].

\section{REFERENCES}

1. Modlin IM, Sandor A. An analysis of 8305 cases of carcinoid tumour. Cancer 1997;79:813-29.

2. Greco FA, Hainsworth JD. Cancer of unknown primary site. In: Devita VT, Hellman A, eds. Cancer-Principles and practice of Oncology. 5th ed. Rosenberg: Lippin Raven 1997:2423-43.

3. Macdonald J, Haller D, Weigel RJ. Endocrine system. In: Adeloff MD, Armitage JO, Lichter AS, Niederhuber JE, eds. Clinical Oncology. 1st ed. New York, Churchill Livingstone Inc, 1995;1047-82.

4. Muir C. Cancer of unknown primary site. Cancer 1995;75(1): 353-6.

5. Thomas RM, Sobin LH. Gastrointestinal cancers. Cancer 1995;75:154-70.

6. Godwin JD. Carcinoid tumours. An analysis of 2837 cases. Cancer 1975;36:560-69.

7. Wynick D, Polak JM. Bloom SR. Neuroendocrine gastroenteropancreatic tumours. In: Peckham M, Pinedo HM, Veronesi U, eds. Oxford text book of Oncology. 1st ed. New York, Oxford Medical Publications, 1995;2145-52. 